REVISIONES

Rev Obstet Ginecol Venez. 2021; 81 (1): 61-74

https://doi.org/10.51288/00810110

\title{
Preparación de un manuscrito para su presentación a una revista científica
}

\author{
Mireya González Blanco. ${ }^{1}$
}

\begin{abstract}
RESUMEN
Publicar el producto del trabajo cientifico es un arte y una necesidad. Si los resultados de una investigación no son informados mediante una publicación en una revista cientifica, los investigadores pierden la oportunidad de que se conozca su trabajo. La preparación del manuscrito es un reto, particularmente para los investigadores que inician su experiencia en la publicación cientifica. Cada revista tiene sus normas de publicación disponibles para los autores, sin embargo, la mayoría de las publicaciones biomédicas siguen las recomendaciones del Comité Internacional de Editores de Revistas Médicas. El presente documento describe la importancia del proceso de investigación, cada una de sus partes y los diferentes tipos de documentos científicos, pero se centra en la descripción de artículos originales, destacando las recomendaciones prácticas para su correcta realización, y presenta los errores que frecuentemente se observan con la intención de guiar a investigadores noveles a través de todo el proceso.
\end{abstract}

Palabras clave: Artículo científico, Publicación médica, Investigación, Recomendaciones para publicar, Artículos originales.

\section{SUMMARY}

Publishing the product of scientific work is an art and a necessity. If the results of the research are not reported through publication in a scientific journal, researchers miss the opportunity for their work to be known. Preparation of the manuscript is a challenge, particularly for researchers who begin their experience in scientific publication. Each journal has its publication standards available to authors; however, most biomedical publications follow the recommendations of the International Committee of Medical Journal Editors. This document describes the importance of the research process, each of its parts and the different types of scientific documents, but focuses on the description of original articles, highlighting practical recommendations for their proper realization, and presents the most frequent errors observed with the intention of guiding novice researchers throughout the process.

Keywords: Scientific paper, Medical publication, Research, recommendations to publish, Original articles.

\section{INTRODUCCIÓN}

La publicación científica es la etapa final de un proceso. Este proceso se inicia con el planteamiento de una idea, le sigue la planificación o elaboración del proyecto, el desarrollo de la investigación en sí misma $\mathrm{y}$, finalmente, la presentación de los resultados.

${ }^{1}$ Directora/Editora de la Revista de Obstetricia y Ginecología de Venezuela.
La idea surge a partir de muchas fuentes. Los problemas aparecen en la práctica clínica diaria, en el ejercicio de actividades docentes, en la lectura de documentos publicados por otros investigadores. Surge, además, en respuesta a problemas nuevos, institucionales, locales, nacionales o internacionales, por ejemplo, la inmensa cantidad de investigación surgida en respuesta a la pandemia por el SAR-CoV-2. Pero también un aumento en la tasa de infecciones en una institución o una inusual elevación en la frecuencia de una patología, puede generar la necesidad de investigar sobre sus causas. Así mismo, el comportamiento de ciertos pacientes frente a diferentes patologías o la respuesta

Vol. 81, № 1, marzo 2021. 
ante diferentes tratamientos, son motivos más que inspiradores para iniciar el camino que lleva a obtener una conclusión válida en el contexto del estudio $y$, de ser científicamente bien conducida, aplicable también a la población general.

La planificación es de gran importancia. Muchas veces se inicia una investigación con una idea clara, pero sin un proyecto elaborado previamente y, llegado a un punto, los investigadores se percatan de que alguna técnica no fue la más adecuada, de que las muestras o los reactivos fueron insuficientes o que los costos no pudieron ser cubiertos y deben incluirse modificaciones, lo que representa una gran pérdida de tiempo, trabajo, recursos humanos (individuos incluidos que deben excluirse por no cumplir con las nuevas estrategias) y económicos. Con frecuencia se da el caso de que una vez finalizado el estudio, el asesor estadístico, consultado tardíamente, señala errores de diseño y la investigación no sirve para los efectos que se había planteado. Por otro lado, la decisión sobre el apoyo científico y económico para el desarrollo de la investigación se sustenta en el proyecto. De allí que la planificación es parte básica del proceso de investigación científica y el investigador, sobre todo aquel que se inicia en este camino, debe asesorarse científica, metodológica, estadísticamente.

El desarrollo de la investigación, depende de la investigación misma, el problema planteado, sus objetivos y el diseño o tipo de estudio. Esta revisión no pretende desarrollar este punto, porque cada investigación y cada investigador seguirán caminos diferentes según los aspectos señalados.

Finalmente, llega la presentación o informe de los resultados. Esta parte es muchas veces descuidada por investigadores clínicosquienes olvidanque la investigación que no se informa, que no se publica, no existe. A pesar de lo importante que pueda ser, si la información obtenida y las conclusiones no están al alcance de la comunidad científica, aunque sea duro decirlo, es tiempo perdido.
Las principales motivaciones que llevan a un científico a publicar sus experiencias y resultados son: establecer una carrera académica (a los hombres de ciencia se les conoce, o no, por sus publicaciones); para el currículum del investigador en busca de trabajo; como intercambio de resultados de investigación y colaboración internacional; como obligación impostergable propia de las carreras y profesiones científicas: lo que no está escrito... no está hecho; la piedra angular en ciencia es que las investigaciones originales tienen que publicarse: para verificarse (reproducibilidad) y para aceptarse e ingresar al conocimiento científico. No solo hay que hacer ciencia, sino escribir ciencia (1).

En este documento se presentará información básica con relación a la publicación de artículos científicos. La base general sobre la que se sustenta la misma es el documento del Comité Internacional de Editores de Revistas Médicas (2), pero va un poco más allá porque incluye información obtenida de múltiples artículos publicados al respecto.

\section{CARACTERÍSTICAS DE UN ARTÍCULO CIENTÍFICO}

Los materiales publicados en revistas pueden clasificarse en una variedad de categorías. Aunque su contenido puede verse influenciado en gran medida por el tipo de material enviado a la revista, el editor es el principal responsable de los tipos de artículos que se publicarán, teniendo en cuenta la necesidad de una mezcla equilibrada. Los tipos de artículos también dependen de la política editorial y de la misión y el alcance de la revista individual. Los distintos tipos de publicaciones son artículos originales, informes de casos, notas técnicas, ensayos gráficos, revisiones narrativas, revisiones sistemáticas, comentarios, editoriales, cartas al editor y material no científico (3).

Los artículos originales son las publicaciones más importantes. Proporcionan nueva información basada en la investigación. Pueden dividirse en artículos 
principales $\mathrm{u}$ originales e informes originales. Los primeros suelen ser prospectivos, están respaldados por un análisis estadístico en profundidad y las conclusiones están apoyadas por los datos proporcionados en los resultados. El informe original es una descripción específica de las observaciones originales sobre las características únicas de una enfermedad o trastorno. Esto puede referirse a un hallazgo diagnóstico previamente descrito, procedimiento o manifestación de una enfermedad. A diferencia de un documento principal, el informe original suele ser retrospectivo y no está sujeto a un análisis estadístico en profundidad (3). El presente documento se refiere a los artículos originales.

Los manuscritos científicos deben estar bien organizados, presentando métodos, datos, análisis e interpretación de una manera clara y comprensible. Un manuscrito científico bien escrito responderá a una importante pregunta de investigación científica de la manera más breve y concisa posible. Por estas razones, se han establecido directrices y enfoques organizativos estructurados para la presentación de informes sobre estudios por imágenes (4). Las características fundamentales de un artículo científico son claridad, precisión y brevedad. Además, los resultados que se presentan deben ser fidedignos y válidos (5).

La aceptación de un artículo científico se basa en cuatro criterios que deben cumplirse: credibilidad, originalidad, claridad y relevancia para los lectores de la revista. Un artículo científico debe incluir cuatro componentes, reconocidos en la forma de las $4 \mathrm{~S}$ (subject, strategy, structure y style). Mientras que el tema y la estrategia forman el trasfondo, la estructura y el estilo representan la forma (6).

El texto de los artículos que informan de una investigación original se divide generalmente en secciones: introducción, métodos, resultados y discusión. Esta estructura llamada IMRyD no es un formato de publicación arbitrario, sino un reflejo del proceso de descubrimiento científico $(1,2,5)$. A este esquema general se agrega el resumen, al inicio, y las referencias, al final. Esta estructura facilita la lectura sectorial/modular ya que los lectores generalmente no leen de manera lineal, sino que navegan por cada módulo en busca de información específica que normalmente existe en secciones preestablecidas. De hecho, cada sección es una respuesta a una pregunta bien codificada (6). El formato IMRyD no es más que un sistema para organizar un trabajo científico, y consiste en responder 4 preguntas claves: (1)

Introducción: ¿Cuál es el problema?

Métodos: ¿Cómo se estudió el problema?

Resultados: ¿Qué se encontró?

Discusión: ¿Qué significan dichos hallazgos?

Puede ser que los artículos requieran dividir esas secciones para organizar aún más su contenido, sin embargo, no es lo habitual. El formato general de otros tipos de artículos, como los metaanálisis, las revisiones, la presentación de casos clínicos y los editoriales puede diferir, siendo, por lo general menos estructurados (2).

Los autores deben definir a qué revista enviarán inicialmente su artículo: esta elección es muy personal y por lo general se apoya en varios criterios. Entre ellos cabe citar la concordancia entre el tema y enfoque del artículo y el ámbito o especialidad de la revista, su alcance nacional o internacional, la reputación o prestigio de la revista, el idioma de publicación, el carácter indexado o no en bases de datos internacionales y su grado y medios de diseminación, su condición de publicación de acceso libre o restringido, el público destinatario principal y la rapidez de publicación. 
Una vez decidida la revista a la que se enviará el documento, los autores deben revisar las instrucciones para los autores; todas las revistas serias incluyen una sección con esta información y conocerla de antemano ayudará a producir un mejor original, con mayores posibilidades de ser aceptado por la revista elegida $(7$, $8)$.

El primer paso en la preparación de cualquier manuscrito es articular el propósito del estudio, el cual debe ser capturado en una sola oración y debe incluirse al principio del resumen y al final de la introducción. Además, se debe reiterar en el primer párrafo de la discusión. Sin un propósito bien definido, el resto del manuscrito será difícil de organizar (4).

Entre los puntos clave que se deben considerar para la escritura de un informe científico para publicación se mencionan los siguientes: los errores en la preparación de manuscritos científicos conducen a su rechazo; la escritura científica, como cualquier habilidad importante, se puede aprender; un enfoque bien desarrollado mejorará la calidad de la escritura científica; la escritura científica de alta calidad es esencial para comunicar los resultados de la investigación; un manuscrito bien organizado comunica más eficazmente un estudio científico (4). Se recomienda que no se utilice la primera persona para la redacción de los informes científicos.

\section{Directrices de presentación de informes}

Se han elaborado directrices de presentación de informes para diferentes diseños de estudio; ejemplos incluyen CONSORT (Consolidated Standards of Reporting Trials) para ensayos aleatorios (9), STROBE (Strengthening the Reporting of Observational Studies In Epidemiology) para estudios observacionales (10), PRISMA (Preferred Reporting Items for Systematic Reviews and Meta-Analyses) para revisiones sistemáticas y metaanálisis (11), y STARD para estudios de precisión diagnóstica (Standards for the Reporting of Diagnostic Accuracy Studies) (12).

El producto principal de CONSORT es la declaración o enunciado CONSORT 2010, que es un conjunto mínimo de recomendaciones basado en evidencia para la notificación de ensayos aleatorios. Ofrece una forma estándar para que los autores preparen informes de los resultados del ensayo, facilitando la presentación de informes completa y transparente y ayudando a su evaluación e interpretación críticas. El enunciado CONSORT comprende una lista de comprobación de 25 elementos y un diagrama de flujo. Los elementos de la lista de verificación se centran en informar cómo se diseñó, analizó e interpretó el ensayo; el diagrama de flujo muestra el progreso de todos los participantes a través de la prueba (9)

Gran parte de la investigación biomédica es de tipo observacional. Los informes de los estudios observacionales a menudo poseen una calidad insuficiente, lo que dificulta la evaluación de sus fortalezas y debilidades para generalizar los resultados. Teniendo en cuenta la evidencia empírica y consideraciones teóricas, un grupo de expertos en metodología, investigadores y editores de revistas científicas, desarrollaron una lista de recomendaciones para aumentar la calidad de las publicaciones de los estudios observacionales: Strenghtening the Reporting of Observational Studies in Epidemiology (STROBE). La Declaración STROBE consiste en una lista de verificación de 22 puntos que guardan relación con las diferentes secciones de un artículo. De ellos, 18 puntos son comunes a los tres diseños de estudio: cohorte, casos y controles, y transversales; los otros cuatro son específicos para cada una de estas tres modalidades. La Declaración STROBE proporciona a los autores información sobre cómo mejorar la calidad de los artículos sobre estudios observacionales y facilita a los revisores, editores de revistas y lectores su apreciación crítica y su interpretación (10). 
PRISMA es un conjunto mínimo de elementos basados en evidencia para la presentación de informes en revisiones sistemáticas y metaanálisis. PRISMA se centra en la presentación de informes de revisiones que evalúan ensayos aleatorios, pero también puede utilizarse como base para informar de revisiones sistemáticas de otros tipos de investigación, en particular evaluaciones de intervenciones. La declaración PRISMA consta de una lista de comprobación de 27 elementos y un diagrama de flujo de cuatro fases. El objetivo de la declaración PRISMA es ayudar a los autores a mejorar la presentación de informes de revisiones y metaanálisis sistemáticos. PRISMA también puede ser útil para la evaluación crítica de revisiones sistemáticas publicadas. Sin embargo, la lista de verificación PRISMA no es un instrumento de evaluación para medir la calidad de un examen sistemático (11).

Los informes incompletos se han identificado como una fuente importante de residuos evitables en la investigación biomédica. La información esencial, a menudo, no se proporciona en los informes de estudio, lo que impide la identificación, la evaluación crítica y la replicación de los estudios. Para mejorar la calidad de la presentación de informes de estudios de precisión diagnóstica, se desarrollaron las Normas para la Notificación de Estudios de Precisión Diagnóstica conocido como STARD. El STARD 2015 es una lista actualizada de 30 elementos esenciales que deben incluirse en cada informe de un estudio de precisión diagnóstica. Esta actualización incorpora evidencia reciente sobre fuentes de sesgo y variabilidad en la precisión del diagnóstico y está destinada a facilitar el uso de STARD. Como tal, STARD 2015 puede ayudar a mejorar la integridad y transparencia en la presentación de informes de estudios de precisión diagnóstica (12).

Se espera que las revistas pidan a los autores que sigan estas pautas porque ayudan a los autores a describir el estudio con suficiente detalle para que sea evaluado por los editores, revisores, lectores y otros investigadores que evalúan la literatura médica. Se alienta a los autores de manuscritos de revisión para describir los métodos utilizados para localizar, seleccionar, extraer y sintetizar datos; esto es obligatorio para revisiones sistemáticas (2).

\section{Secciones de manuscrito}

Los siguientes son requisitos generales para la presentación de informes dentro de las secciones de todos los diseños de estudio y formatos manuscritos.

Página de título

La información general sobre un artículo y sus autores se presenta en una página de título del manuscrito y por lo general incluye el título del artículo, la información del autor, cualquier descargo de responsabilidad, fuentes de apoyo, recuento de palabras y, a veces el número de tablas y figuras (2).

Título del artículo. El título proporciona una descripción del artículo completo y debe incluir información que, junto con el resumen, hará que la recuperación electrónica del artículo sea sensible y específica. Las pautas de presentación de informes recomiendan y algunas revistas requieren que la información sobre el diseño del estudio sea parte del título (especialmente importante para ensayos aleatorios y metaanálisis) (2).

Su extensión debe tener la menor cantidad de palabras posibles que describan los contenidos del trabajo (10-12 palabras), con efectividad en la sintaxis y sin requerimientos gramaticales fuertes, esto es, claro y conciso (1). No debe incluir fórmulas, nombres patentados, acrónimos, siglas, nombres poco comunes, ni abreviaturas. Tiene que ser autoexplicativo y evitar palabras inespecíficas que no aportan significado (5). Es recomendable definir un título tentativo previo a redactar el manuscrito y elaborar el título final luego 
de terminar el artículo (1). A diferencia del objetivo del estudio, el título no se debe delimitar en tiempo, espacio y persona. Además, es conveniente que incluya las variables principales que se investigan.

Entre los errores más frecuentes que se observan al revisar los títulos de los artículos científicos están los siguientes: títulos demasiados largos, poco atractivos, que no concuerdan con el tema o el objetivo, incomprensibles, redundantes, incompletos, con abreviaturas o siglas y con errores de ortografía (13).

Información del autor. El título académico más alto de cada autor debe incluirse, aunque algunas revistas no lo publican. El nombre del (los) departamento (s) e institución (es) u organizaciones en las que el trabajo se desarrolla debe especificarse. También es importante que los autores proporcionen información de contacto completa, incluyendo el correo electrónico. El Comité Internacional de Editores de Revista Médicas (ICMJE) estimula a incluir el listado de la identificación abierta de investigadores y colaboradores de los autores (ORCID) (2). ORCID proporciona un identificador digital persistente que posee y controla el investigador y que lo distingue de cualquier otro investigador. Esta identificación se puede conectar con la información profesional: afiliaciones, subvenciones, publicaciones, reseñas por pares y mucho más. Se puede utilizar para compartir información con otros sistemas, asegurando que se obtenga reconocimiento por todas las contribuciones, lo que ahorra tiempo y molestias y reduce el riesgo de errores (14)

Descargos de responsabilidad. Un ejemplo de descargo de responsabilidad es la declaración de que las opiniones expresadas en el artículo presentado son propias y no una posición oficial de la institución o financiador (2).

Fuente(s) de soporte. Estos incluyen subvenciones, equipos, medicamentos y/u otro apoyo que facilitara la conducción del trabajo descrito en el artículo o la escritura del artículo en sí mismo (2).

Conteo de palabras. Las revistas pueden solicitar un recuento de palabras para el texto del documento, excluyendo sus resúmenes, reconocimientos, tablas, leyendas de figuras y referencias, este permite a editores y revisores evaluar si la información contenida en el documento justifica la longitud del documento y si el manuscrito se ajusta a los formatos de la revista. Un recuento de palabras separado para el resumen es útil por la misma razón (2).

\section{Resumen}

El propósito del resumen es despertar el interés del lector por la lectura total del artículo (1).

Los artículos originales, las revisiones sistemáticas y los metaanálisis requieren resúmenes estructurados. Esto es, requieren que se incluyan secciones, claramente definidas e identificadas: objetivo, métodos, resultado y conclusión. El resumen debe proporcionar el contexto o antecedentes para el estudio (algunas revistas no incluyen esta introducción) y debe definir el propósito del estudio, los procedimientos básicos (tipo de estudio, selección de participantes, mediciones, seguimiento), principales hallazgos (dando tamaños de efectos específicos y su importancia estadística y clínica, si es posible) y principales conclusiones. Debido a que los resúmenes son la única porción sustantiva del artículo indexado en muchas bases de datos electrónicas y que es la única parte que muchos leerán, los autores deben asegurarse de que reflejan con precisión el contenido del artículo (2). El resumen no debe incluir material o información que no esté incluida en el documento completo, no lleva referencias y debe ser redactado en pasado. Es importante enfatizar que el resumen se debe entender por sí solo, sin necesidad de leer el artículo (1). Por regla general, el resumen estructurado consta de un máximo de 250 palabras. En los artículos 
que incluyen resúmenes no estructurados, estos deben contener un máximo de 150 palabras.

Al final del resumen, el autor debe incluir alrededor de cinco palabras clave, que servirán para efecto de la indexación. La selección de palabras clave es sumamente importante porque esta es la manera en la que los motores de búsqueda indexarán el artículo publicado. Si las palabras clave se seleccionan adecuadamente habrá más posibilidades de que el artículo sea encontrado, leído y citado. Para una selección adecuada de las palabras clave se puede utilizar el Índice Médico Español o los Medical Subject Headings (MeSH) del Index Medicus o del Índice Médico Español (5). Las palabras clave se escriben iniciando con mayúscula y separadas por comas.

Los errores frecuentes encontrados en el resumen son la extensión, muy larga o demasiado corta, no sintetiza apropiadamente los aspectos relevantes del estudio, contiene fragmentos copiados y pegados a partir de otras secciones del documento, incluye información que no aparece en el resto del estudio, incluye abreviaturas y referencias o citas (13).

Introducción

En esta sección se debe proporcionar un contexto o antecedentes para el estudio, la naturaleza del problema y su significado. Se incluye el propósito específico u objetivo de investigación. Se debe citar solo referencias pertinentes (ver sección de referencias) y no se debe incluir datos ni conclusiones del trabajo que se está reportando (2). Esta introducción debe ser completa pero no innecesariamente larga, debe incluir información sobre el problema específico sobre el que se investiga y no sobre el tema general. Se redacta en presente, aunque los antecedentes pueden admitir la redacción en pasado y tiene una estructura de lo general a lo particular (7).

Vol. 81, $\mathrm{N}^{\mathrm{o}} 1$, marzo 2021.
Los tres errores principales que se deben evitar en esta sección se refieren a su longitud, el tiempo del verbo y el uso de abreviaturas o siglas no explicadas. En cuanto a la extensión, si el tema es clásico y si el artículo está destinado a una revista especializada, la introducción debe ser breve. Por otro lado, si el tema es poco tratado en la literatura o si el artículo se publicará en una revista no especializada, la introducción debe ser más amplia. En general, no debe representar más del $10 \%$ a $15 \%$ del número total de palabras. En cuanto al tiempo del verbo, tal como se señaló, el tiempo pasado es la regla cuando se informan los resultados de otros estudios, o cuando el autor reporta hallazgos que considera controvertidos. El presente se utilizará para hechos establecidos, es decir, la síntesis de estudios, problemas y contextos similares. Finalmente, el abuso de abreviaturas hace que el texto sea incomprensible (6). También se ha señalado como errores frecuentes, la falta o el exceso de referencias, errores de redacción y el no señalar el objetivo de la investigación (13). No se recomienda el uso de la primera persona para la redacción.

\section{Métodos}

El principio rector de esta sección debe ser claridad sobre cómo se hizo el estudio. Debe ser lo suficientemente detallada para permitir a otros reproducir los resultados. En general, la sección debe incluir solo la información que estaba disponible en el momento en que el plan o protocolo para el estudio estaba siendo escrito; toda la información obtenida durante el estudio se incluye en la sección de resultados. Es recomendable incluir un comentario que indique que la investigación fue aprobada por un organismo de revisión local, regional o nacional (por ejemplo, comité de ética o administración institucional) (2).

La sección de métodos se puede organizar en 5 áreas: diseño, población y muestra (tamaño de la muestra, criterios de inclusión y exclusión, criterios 
utilizados para la distribución en grupos), el entorno, las intervenciones o detalles del procedimiento $\mathrm{y}$ el análisis estadístico (1). Como regla general, esta sección se redacta en pasado.

El diseño debe ser seleccionado adecuado al propósito de la investigación. Todos los diseños no son útiles para todas las investigaciones, este es un error que se observa con mucha frecuencia. Se debe describir claramente la selección de participantes (individuos o pacientes sanos, controles), la técnica de muestreo (probabilístico o intencional), el tamaño de la muestra, los criterios de inclusión y exclusión, criterios utilizados para la distribución en grupos y una descripción de la población de origen. En caso de muestreo aleatorio, se debe explicar el procedimiento: aleatorio simple, aleatorio estratificado, aleatorio por conglomerado o aleatorio sistemático. Si en el muestreo se utilizó el procedimiento no aleatorio, debe explicarse el muestreo de conveniencia, de cuota o intencional, es decir, el tamaño de la muestra se informa a la luz del objetivo del estudio, del diseño, del método de muestreo y del análisis estadístico de los datos (15). Identificar métodos, equipos (dar nombre y dirección entre paréntesis) y los procedimientos en suficiente detalle para permitir que otros reproduzcan los resultados. Si los métodos empleados son conocidos, se deben mencionar y referir, pero cuando no son procedimientos conocidos o se ha introducido alguna modificación, se debe hacer una descripción breve, dar las razones para usarlos y evaluar sus limitaciones. Identificar con precisión todos los medicamentos y productos químicos utilizados, incluyendo nombres genéricos, dosis y ruta(s) de administración (2). También se describen los cuestionarios, escalas de variables e instrumentos que se utilizaron, así como los valores que se consideran normales durante la investigación. Cada cuestionario debe tener una breve descripción indicando los factores que mide. Si hubiera subescalas, únicamente se deben enumerar si la información es relevante para el artículo (5).
En la sección de métodos debe incluirse comentarios sobre los aspectos bioéticos pertinentes, mencionar el consentimiento informado en investigaciones prospectivas y la confidencialidad y anonimato en estudios tanto prospectivos como retrospectivos.

Los métodos estadísticos se deben describir con suficiente detalle para que un lector experto con acceso a los datos originales pueda juzgar su idoneidad para el estudio y verificar los resultados reportados. Cuando sea posible, se debe cuantificar los hallazgos y presentar indicadores adecuados de medición de error o incertidumbre (como intervalos de confianza). Definir términos estadísticos, abreviaturas y la mayoría de los símbolos. Especificar los paquetes de software estadístico y las versiones utilizadas (2).

Los tres errores relacionados con esta sección de métodos, se refieren a su extensión, la presentación de resultados y el uso de verbos. La longitud de la sección no debe exceder el $30 \%$ del artículo (ideal: $20 \%$ a $30 \%$ ). Por esta razón, no se recomienda sobrecargar esta sección con detalles innecesarios. No se recomienda el informe de resultados en esta sección. Con respecto al tiempo del verbo, el autor debe elegir el tiempo pasado (imperfecto o compuesto) para describir lo que se ha definido y logrado en el estudio. El tiempo presente solo es aceptable para indicar que una técnica es estándar o que una herramienta de medición es confiable/válida (6). También se señalan como errores la elección incorrecta del tipo de estudio, la omisión de la delimitación (tiempo y lugar), la falla en reportar los aspectos ya descritos que deben formar parte de la sección, uso de instrumentos no validados u obsoletos, no se establecen los puntos de corte o los valores considerados normales, falta de control o presencia de sesgos (13).

Resultados

Los resultados se deben presentar en una secuencia 
lógica en el texto, dando primero los resultados principales o más importantes. Es requisito incluir los números absolutos a partir de los cuales se calcularon las frecuencias relativas. Las tablas, figuras o gráficos se incluyen en anexos, numeradas según el orden de aparición, y deben ser referidos en el texto. No se debe repetir todos los datos de las tablas o figuras en el texto; enfatizar o resumir solo la mayoría de las observaciones importantes. Restringir tablas y figuras a las necesarias para explicar el objetivo de la investigación y para evaluar los datos de apoyo. Cada revista establece el número máximo de tablas a ser incluidas, de forma general, se recomiendan entre 2 y 6 tablas, pero idealmente es más adecuado incluir entre 3 y 4 tablas (5). Se pueden utilizar gráficos como alternativa a las tablas con muchas entradas pero no duplicar datos en gráficos y tablas (2). Una figura incluye todo tipo de material no tabular (morfología, algoritmos, histogramas, gráficas, fotografías, etc.). La tabla tiene la ventaja de mostrar mejor los valores numéricos exactos con sus posibles interrelaciones, mientras que un gráfico expresa mejor la tendencia de los datos o patrones bien definidos, es decir, describe una situación que cambia en el tiempo o en el espacio; también son útiles al hacer comparaciones. Por tanto, la tabla se utilizará cuando la precisión de los datos es importante y el gráfico cuando los datos presentan una tendencia definida o permiten resaltar una diferencia $(1,5,6)$.

Las recomendaciones prácticas para esta sección incluyen: redacción sencilla y breve, siempre hacer referencia a la tabla, figura o gráfico que se describe, salvo excepciones, se recomienda separar los resultados en párrafos diferentes, no incluir análisis de los resultados ni juicios personales en esta sección, incluir la información estadística relevante, sin explicarla. Se redacta en pasado. En líneas generales, a pesar de ser una sección de gran importancia en el documento, también debería ser la sección más breve.
Los principales errores relativos incluyen problemas de estilo, extensión del texto y uso indebido de ilustraciones. Los problemas de estilo más frecuentes se refieren a la no utilización del tiempo pasado, el incumplimiento de la obligación de un tono neutro y especialmente la falta de precisión. Como ya se mencionó, los resultados deben presentarse sin interpretación. En términos de extensión, sin incluir las ilustraciones, esta sección debe ser una sección corta. De hecho, su longitud habitual es aproximadamente un $10 \%$ a $20 \%$ de la del artículo. El mal uso de ilustraciones es un error común. Muchas ilustraciones son incomprensibles independientemente del texto. Las ilustraciones deben ser autoexplicativas y el lector debe poder entender completamente la información sin tener que leer el texto. Muchos artículos científicos tienen redundancia entre texto e ilustraciones. El autor no debe repetir en el texto toda la información contenida en las ilustraciones sino solo destacar los resultados que corroboran la hipótesis y los resultados inesperados (6). También se describen como errores, mencionar resultados no relacionados con los objetivos, pruebas estadísticas incorrectas, los números absolutos no se acompañan de porcentajes, la presencia de errores en las cifras, la inclusión de referencias y la falta de unidades de medida (13).

\section{Discusión}

La discusión es la parte central del documento. Los resultados por sí solos pueden no aportar toda la información requerida, a menos que sean correctamente interpretados. Aquí el investigador puede dar rienda suelta a su capacidad de análisis.

Es útil comenzar el debate resumiendo brevemente el propósito del estudio y los principales hallazgos y explorar posibles mecanismos o explicaciones para estos hallazgos. Se recomienda hacer hincapié en lo nuevo y en los aspectos importantes del estudio y poner sus hallazgos en el contexto de la totalidad de 
las pruebas pertinentes. Describir las limitaciones del estudio y explorar las implicaciones de los hallazgos para futuras investigaciones y para la práctica clínica. Discutir la influencia o asociación de variables, cuando sea apropiado. No repetir en detalle datos $u$ otra información proporcionada en otras partes del manuscrito, como en la introducción o resultados. Vincular las conclusiones con los objetivos del estudio, pero evitar declaraciones y conclusiones no apoyadas en los resultados (2).

Las recomendaciones para la redacción de la discusión incluyen lo siguiente: se debe redactar en presente. Evitar repetir los resultados a menos que un dato sea indispensable para su análisis, comparar los resultados con investigaciones previas, aunque esto no es indispensable ni suficiente; no tiene valor decir que un resultado es igual o diferente al de una investigación previa si no se analizan las razones. Se puede especular y teorizar en forma lógica y relacionar los resultados con los objetivos planteados. Así mismo, mencionar los resultados inesperados y analizarlos y, finalmente, plantear la necesidad de investigaciones futuras, en caso que los resultados de la investigación así lo señalen. Plantear aquí las fortalezas y limitaciones del estudio, siendo recomendable señalar como se superaron o controlaron dichas limitaciones. Al finalizar la discusión se incluirán las conclusiones y recomendaciones. Respecto a ellas, es muy importante que las mismas deban derivar de la investigación y no de la revisión de la literatura científica realizada. Las conclusiones pueden redactarse en forma corrida o pueden enumerarse, pero siempre deben relacionarse con los objetivos de la investigación.

Los principales errores relacionados con esta sección se refieren a la extensión, la autocrítica, la revisión de la literatura, el tiempo verbal y la modestia. La longitud ideal de la sección es el $50 \%$ del artículo. Una discusión demasiado larga hace que la sección sea difícil de organizar pero si es demasiado corta da la impresión de lagunas de conocimiento. La extensión excesiva puede deberse a repeticiones innecesarias, introducción de nuevos resultados para sustentar argumentos, descripción de métodos para argumentar la validez, enfoque no sintético durante la etapa de comparación, con una revisión en profundidad de la literatura. La autocrítica es un elemento esencial. Algunos autores destacan que han enumerado todos los sesgos posibles y los eliminaron, en cambio, otros optan por la enumeración de todos los detalles relacionados con las dificultades metodológicas, hasta el punto de que el lector podría cuestionar la validez de los resultados. Algunos autores escriben la discusión como si fuera una revisión de la literatura, sin ánimo de sintetizar los datos y otros no actualizan sus referencias. El cuarto error relacionado con esta sección se refiere al tiempo del verbo. En la práctica, se debe utilizar el pasado para evocar el estudio o para describir los resultados de otros estudios, el presente para evocar los hechos, conceptos y teorías establecidas, el futuro para evocar las perspectivas y el condicional para adelantar las hipótesis. La falta de modestia es un error inaceptable. El autor no debe exagerar ni la originalidad del estudio, el rigor de los métodos, la importancia de los resultados ni la abundancia de referencias bibliográficas (6). Otros dos errores frecuentes en la discusión son reportar nuevamente los resultados y discutir aspectos que no se han presentado en estos (7). En la conclusión, los errores que se observan con más frecuencia tienen que ver con la inclusión de conclusiones muy extensas o muy breves, no relacionadas con los objetivos de la investigación (13).

\section{Agradecimiento}

En la sección de agradecimientos se ha de reconocer el trabajo y la colaboración tanto de personas como de instituciones que hayan ayudado en la redacción y revisión del artículo. En esta sección también se enumera la financiación (becas o ayudas) recibida durante el periodo en que se ha realizado la investigación y/o se ha redactado el manuscrito (5). El agradecimiento es exclusivo para personas que hayan 
participado directamente en la investigación motivo de la publicación.

Después del agradecimiento, se incluye una declaración de conflicto de interés (7), en caso que esta no se haya incluido en la página del título.

\section{Referencias}

Las referencias cumplen dos funciones esenciales: testificar y autentificar los datos no originales del trabajo y proveer al lector de literatura referente al tema en cuestión. Las revistas varían mucho en la forma de tratar las referencias. Existen múltiples estilos para incluir las referencias en el texto y para redactar las referencias en la lista al final del manuscrito. La cita en el texto puede hacerse incluyendo el nombre del autor y el año de la cita entre paréntesis, asignando números según un orden alfabético o asignando números según el orden de aparición (1). La mayoría de las publicaciones científicas de índole médica o biomédica utilizan el estilo de redacción de las referencias según el Comité Internacional de Editores de Revistas Médicas (2) y este documento se refiere particularmente a ese estilo.

Los autores deben proporcionar referencias directas a fuentes de investigación siempre que sea posible. Las referencias no deben ser utilizadas por autores, editores o revisores de pares para promover autointereses. Las referencias a documentos aceptados pero aún no publicados deben ser designadas como "en prensa" o "próximamente." La información de manuscritos presentados pero no aceptados debe citarse en el texto como "observaciones inéditas" con permiso por escrito de la fuente. Se debe evitar citar una "comunicación personal" a menos que proporcione información esencial no disponible de una fuente pública, en cuyo caso el nombre de la persona y la fecha de comunicación deben citarse entre paréntesis en el texto, además, el autor debe obtener permiso y confirmación por escrito de la fuente de una comunicación personal. Algunas revistas, pero no todas, comprueban la exactitud de todos los citas de referencia; por lo tanto, los errores de cita a veces aparecen en la versión publicada de los artículos. Para minimizar estos errores, las referencias deben verificarse utilizando un indizador bibliográfico, como PubMed, o copias impresas de fuentes originales. Los autores son responsables de comprobar que ninguna de las referencias citan artículos retractados, excepto en el contexto de referencia a la retractación (2).

Las referencias deben numerarse consecutivamente en el orden en el que se mencionan por primera vez en el texto, tablas y/o leyendas, por números arábigos, pero cada revista establecerá su estilo, por lo general entre paréntesis, puede ser en superíndice o a nivel del texto. Cuando se cita más de una referencia en forma consecutiva, se incluye la primera y la última, separadas por un guion. Si las referencias no son consecutivas se separan por comas. Si hay más de un autor y es necesario nombrarlo en el texto, se señala solo el apellido del primer autor, acompañado de la expresión et al. (en latín); algunas revistas prefieren la expresión y cols., en ambos casos, seguido de la referencia entre paréntesis. En la lista de referencias, se debe citar los autores (apellido e inicial del nombre), hasta un máximo de 6. En caso de ser más de seis autores, se deben anotar los seis primeros y luego et al. Todas las referencias colocadas en la lista final deben estar incluidas en el texto. Los autores de las referencias citadas en el texto deben coincidir con los autores de las referencias de la lista final.

Las referencias deben seguir las normas resumidas por el Comité Internacional de Revistas Médicas (ICMJE) $(16,17)$. Estos recursos son actualizados regularmente a medida que se desarrollan nuevos medios y actualmente incluyen orientación para documentos impresos; material inédito; audio y medios visuales; material en CD-ROM, DVD o disco; y material en Internet (2). 
Se han observado inexactitudes en las referencias de muchos artículos publicados. Los principales errores están relacionados con la confusión entre el nombre y apellido de los autores, títulos falsos del documento, numeración incorrecta, errores en el título de la revista, en el número de volumen, en el año de publicación y en el número de página (6). Otros errores señalados con frecuencia son el uso de referencias desactualizadas cuando existen investigaciones recientes y la inclusión de resúmenes como referencias (13).

\section{Tablas}

Las tablas presentan la información de forma concisa y eficiente; también proporcionan información en cualquier nivel de detalle y precisión. Incluir datos en tablas en lugar del texto con frecuencia hace posible reducir la longitud del documento. Se deben preparar según el formato establecido por cada revista. Se numeran en forma consecutiva según su primera mención en el texto. Los títulos de las tablas deben ser cortos pero deben contener información que permita a los lectores entender el contenido de la tabla sin tener que volver al texto (2).

Las tablas no deben llevar líneas internas, excepto las que separan los encabezados de filas y columnas. Se debe evitar el uso de abreviaturas en las tablas, pero cuando sea indispensable, se explicarán en notas al pie de página. También se deben identificar las medidas estadísticas de variaciones, como desviación y error estándar de la media. Las tablas que incluyen los resultados de la investigación no llevan fuente.

\section{Ilustraciones (Figuras)}

Las imágenes digitales de ilustraciones manuscritas deben estar en un formato adecuado para la publicación impresa. Para presentar copias impresas, las figuras pueden ser dibujadas profesionalmente y fotografiadas o enviadas como impresiones digitales con calidad fotográfica. Para imágenes radiológicas y otras imágenes clínicas y diagnósticas, así como imágenes de especímenes de patología o fotomicrografías, se debe enviar una imagen fotográfica de alta resolución. Las letras, los números y los símbolos de las figuras deben ser claros y consistentes en todo, y lo suficientemente grandes como para permanecer legible cuando la imagen se reduzca para su publicación. Los títulos y explicaciones detalladas se ubicarán como leyenda y no sobre la figura en sí. Si una figura se ha publicado anteriormente, se debe incluir la fuente original y obtener permiso por escrito para reproducirlo. Se requiere permiso, independientemente de la autoría o el editor, excepto en caso de documentos de dominio público (2). Las figuras y los gráficos que incluyen los resultados de la investigación no llevan fuente.

Para finalizar este artículo, es importante considerar algunos aspectos para mejorar la redacción del documento que se envía para publicación. Aunque la investigación sea excelente y el informe final cumpla con todos los pasos señalados, puede que el autor no logre transmitir lo que desea si no redacta en forma adecuada. Algunos errores e imprecisiones del lenguaje en la redacción de artículos científicos fueron señalados por la autora en una publicación previa (18). De forma general, es recomendable usar el diccionario de la Real Academia de la Lengua (19), fácilmente disponible en línea, para utilizar los términos más adecuados, nunca utilizar primera persona en la redacción de artículos originales, evitar los modismos y los anglicismos, así como también el uso indiscriminado de gerundios y mayúsculas, redactar de forma sencilla, sin rebuscar palabras que restan claridad a lo que se quiere expresar, y utilizar oraciones cortas. Se deben explicar las siglas la primera vez que se utilizan y hacer uso adecuado de los signos de puntuación. También es preferible evitar párrafos muy largos que dificultan la lectura o la hacen tediosa. Una revisión ortográfica exhaustiva debe hacerse antes de entregar el manuscrito. 
En relación al formato de presentación del artículo, cada revista tiene sus normas y, generalmente, están disponibles en su página web o dentro de la revista misma. Sin embargo, de forma general, se recomienda configurar la computadora para trabajar en formato Word, tamaño carta, fondo blanco y letra negra; usar una letra sencilla, clara, sin adornos (Times New Roman o Arial), tamaño 12, con márgenes adecuados (entre dos y tres centímetros por lado), con interlineado amplio (1,5 o 2 líneas) y marcando los párrafos con un espaciado ligeramente superior al interlineado.

Antes de enviar el manuscrito, se debe revisar de manera sistemática, verificar si el manuscrito es claro, preciso, conciso y completo. Comprobar si la información es coherente, sin contradicciones internas, y que cada elemento está donde debe estar. Verificar si las figuras y los cuadros son autoexplicativos y claros y están citados en el texto. El autor debe cerciorarse de que la redacción (ortografía, puntuación, terminología) es correcta. Por último, verificar que el manuscrito cumple las instrucciones de la revista (7).

\section{CONCLUSIONES}

La publicación de un informe científico es la etapa final del proceso de investigación, indispensable para dar a conocer los resultados de la misma. Para muchos autores, la presentación de ese informe ante los comités editoriales de las revistas suele ser un reto. En este documento se pretendió brindar orientación relevante para culminar el proceso con éxito. La base fundamental son las recomendaciones del Comité Internacional de Editores (2), pero se incluyó material adicional proveniente de otras fuentes y sugerencias de la autora para complementar la información.

\section{REFERENCIAS}

1. Villagrán A, Harris P. Algunas claves para escribir correctamente un artículo científico. Rev Chil Pediatr [Internet]. 2009 [consultado 18 de octubre de 2020]; 80(1): 70-78. Disponible en: https://scielo.conicyt.cl/scielo.php?script=sci arttext\&pid=S0370-41062009000100010

2. ICMJE. International Committee of Medical Journals Editors [Internet]. Vancouver: Recommendations for the Conduct, Reporting, Editing, and Publication of Scholarly Work in Medical Journals; 2019 [actualizado diciembre de 2019; consultado 18 de octubre de 2020]. Disponible en: http://www.icmje.org/ recommendations/

3. Peh WCH, Ng KH. Effective medical writing. Singapore Med J [Internet]. 2008 [consultado 18 de octubre de 2020]; 49(7):522-524. Disponible en: http://www.smj. org.sg/sites/default/files/5101/5101emw1.pdf

4. Bannas P, Reeder SB. How to write an original radiological research manuscript. Eur Radiol. 2017; 27(11):4455-4460. doi: 10.1007/s00330-017-48798. Epub 2017 Jun 14. PMID: 28616726; PMCID: PMC6599681.

5. Santesteban O, Nuñez N. Cómo escribir un artículo científico por primera vez. Psiq Biol. 2017; 24(1):3-9. doi: 10.1016/j.psiq.2017.01.004

6. Ben Saad H. Scientific Medical Writing in Practice: the «IMR@D®» Format. Tunis Med [Internet]. 2019 [consultado 18 de octubre de 2020]; 97(3):407425. Disponible en: https://pubmed.ncbi.nlm.nih. gov/31729715/

7. Organización Panamericana de la Salud/Organización Mundial de la Salud [Internet]. Washington DC: Cómo empezar a escribir un artículo científico; 2015 [consultado 18 de octubre de 2020]. Disponible en: https://www.paho.org/hq/dmdocuments/2017/gestionconocimiento-Como-escribir-artigo-cientifico.pdf

8. Shidham VB, Pitman MB, Demay RM. How to write an article: Preparing a publishable manuscript! Cytojournal. 2012; 9:1. doi: 10.4103/1742-6413.92545. Epub 2012 Jan 31. PMID: 22363390; PMCID: PMC3280045.

9. CONSORT. Transparent reporting of trials [Internet]. The CONSORT statement; 2010 [consultado 18 de octubre de 2020]. Disponible en: http://www.consortstatement.org/

10. STROBE Statement [Internet]. Berna: Strengthening the Reporting of Observational studies in Epidemiology; 2014 [consultado 18 de octubre de 2020]. Disponible en: http://strobe-statement.org/

11. PRISMA. Transparent reporting of systematic reviews and meta-analyses [Internet]. PRISMA Statement; 2009 [consultado 18 de octubre de 2020]. Disponible en: http://prisma-statement.org/ 
12. Equator network [Internet]. Oxford: STARD 2015: An Updated List of Essential Items for Reporting Diagnostic Accuracy Studies; 2015 [consultado 18 de octubre de 2020]. Disponible en: http://www.equatornetwork.org/reporting-guidelines/stard/.

13. Hernández-Vargas A, Pérez-Manjarrez FE, MendiolaPastrana IR, López-Ortiz E, López-Ortiz G. Errores más comunes al redactar artículos médicos originales. Gac Med Mex. 2019;155(6):635-640. doi: 10.24875/ GMM.19005172. PMID: 31787766.

14. ORCID. Connecting research and researchers [Internet]. What is ORCID?; 2019 [consultado 18 de octubre de 2020]. Disponible en: https://orcid.org/

15. Henríquez E, Zepeda M. Elaboración de un artículo científico de investigación. Ciencia Enf [Internet]. 2004 [consultado 18 de octubre de 2020]; X(1):1721. Disponible en: https://scielo.conicyt.cl/pdf/cienf/ v10n1/art03.pdf

16. National Library of Medicine [Internet]. Bethesda: Samples of formatted references for authors of journal articles; 2010 [consultado 18 de octubre de 2020]. Disponible en: www.nlm.nih.gov/bsd/uniform requirements.html
17. Patrias K, autor, Wendling D editor. Citing Medicine: The NLM Style Guide for Authors, Editors, and Publishers [Internet]. 2nd edition. Bethesda: National Library of Medicine (US); 2007 [consultado 18 de octubre de 2020]. Disponible en: www.ncbi.nlm.nih. gov/books/NBK7256/.

18. González Blanco M. Errores e imprecisiones ortográficas al escribir un artículo científico. Rev Obstet Gin Venez [Internet]. 2019 [consultado 18 de octubre de 2020]; 79(2):58-61. Disponible en: http://www.sogvzla. org.ve/sogvzla $20186 / \mathrm{cms} / \mathrm{svcobtenerpdfrevista.}$ php?id $=0000000094 \&$ tipo $=$ normal\&fila $=2$

19. Real Academia de la Lengua RAE [Internet]. Madrid: Diccionario de la lengua española; 2014 [actualizado 2019; consultado 18 de octubre de 2020]. Disponible en: https://dle.rae.es/contenido/ actualizaci\%C3\%B3n-2019

Recibido 10 de octubre de 2020 Aprobado 20 de noviembre de 2021 